CORRIGENDUM

doi:10.1038/nature09930

\title{
Mediator and cohesin connect gene expression and chromatin architecture
}

Michael H. Kagey, Jamie J. Newman, Steve Bilodeau, Ye Zhan, David A. Orlando, Nynke L. van Berkum,

Christopher C. Ebmeier, Jesse Goossens, Peter B. Rahl, Stuart S. Levine, Dylan J. Taatjes, Job Dekker

\& Richard A. Young

Nature 467, 430-435 (2010)

It is important to note that components of the cohesin and mediator complexes, including Smc1a, Nipbl, Rad21 and Med10, were identified in previous RNA interference screens for maintenance of embryonic stem cells ${ }^{1-3}$.

1. Hu, G. et al. A genome-wide RNAi screen identifies a new transcriptional module required for self-renewal. Genes Dev. 23, 837-848 (2009).

2. Fazzio, T. G., Huff, J. T. \& Panning, B. An RNAi screen of chromatin proteins identifies Tip60-p400 as a regulator of embryonic stem cell identity. Cell 134, 162-174 (2008).

3. Ding, L. etal. A genome-scale RNAi screen for Oct4 modulators defines a role of the Paf1 complex for embryonic stem cell identity. Cell Stem Cell 4, 403-415 (2009). 